\title{
Entropy-Like State Counting Leads to Human Readable Four Color Map Theorem Proof
}

\author{
Robert Luckett Shuler Jr. \\ NASA, Johnson Space Center, Houston, USA \\ Email address: \\ robert@shulerresearch.org \\ To cite this article: \\ Robert Luckett Shuler Jr. Entropy-Like State Counting Leads to Human Readable Four Color Map Theorem Proof. Pure and Applied \\ Mathematics Journal. Vol. 7, No. 3, 2018, pp. 37-44. doi: 10.11648/j.pamj.20180703.12
}

Received: August 24, 2018; Accepted: September 7, 2018; Published: September 28, 2018

\begin{abstract}
The problem of how many colors are required for a planar map has been used as a focal point for discussions of the limits of human direct understanding vs. automated methods. It is important to continue to investigate until it is convincingly proved map coloration is an exemplary irreducible problem or until it is reduced. Meanwhile a new way of thinking about surfaces which hide $\mathrm{N}$-dimensional volumes has arisen in physics employing entropy and the holographic principle. In this paper we define coloration entropy or flexibility as a count of the possible distinct colorations of a map (planar graph), and show how a guaranteed minimum coloration flexibility changes based on additions at a boundary of the map. The map is 4-colorable as long as the flexibility is positive, even though the proof method does not construct a coloration. This demonstration is successful, resulting in a compact and easily comprehended proof of the four color theorem. The use of an entropy-like method suggests comparisons and applications to issues in physics such as black holes. Therefore in conclusion some comments are offered on the relation to physics and the relation of plane-section color-ability to higher dimensional spaces. Future directions of research are suggested which may connect the concepts to not only time and distance and thus entropic gravity but also momentum.
\end{abstract}

Keywords: Graph Theory, Combinatorics, Four Color Map Theorem, Entropy, Gravity, Equivalence Principle

\section{Introduction}

The question of the minimum number of colors with which to color a map so that no adjacent countries sharing a border of non-zero length have the same color has enjoyed popularity since the middle 1800 s when it was introduced by mathematician Augustus De Morgan, it having come to his attention through Francis and Fredrick Guthrie [1]. In 1879-80 well known proofs by Kempe and Tait were published and each stood for eleven years before being discovered to be incorrect. The problem was never of particular concern to actual map makers, but was further popularized in September 1960 by Martin Gardner writing in the Mathematical Games column of Scientific American magazine, followed in 1975 by an April Fools hoax column with a supposed counter-example. The very next year Kenneth Appel and Wolfgang Haken disclosed a computer-generated proof asserting that a counter-example must contain, yet could not contain, one of a particular set of 1,936 maps [2-3]. There was some resistance to acceptance because such a proof could not be reliably checked by hand [4]. There followed a simpler proof still relying on computers in
1997 [5], and a proof using theorem-proving software in 2005, formally published in 2008 [6].

The problem is still interesting. It is an exercise in finding the boundary of the problem space which is approachable by humans, vs. those which must be solved by automation or even artificial intelligence. Its apparent simplicity makes it particularly important if it is found to not be solvable by humans without computer assistance. Thus new proofs continue to appear, attempting to best the previous ones in some aspect.

The author suspects the sub-map approach is a source of complexity and difficulty in human construction and verification and for some time has sought an alternative. The main problem is to guarantee color-ability without constructing a particular coloration. Inductions on coloration, or constructive proofs, i.e. constructing coloration, fail because later steps in the induction sequence, when chosen from among alternatives, invariably require re-coloration, or back-tracking, and since the order backward cannot be carefully selected and controlled as the order forward, the simpler proofs fail. More complex proofs succeed but we humans only understand them abstractly, not in detail. The question of interest in this paper is 
whether this is intrinsic to the 4-color map problem itself, or to the ways in which it has been conceptualized.

We consider a conceptualization in terms of state counting, very much like entropy, and explore whether state counting can be formulated as a single node induction sequence in a way that implements the obvious hiding aspects of planar maps while avoiding the back-tracking problem. A state is, loosely, a particular coloration of the map. If a different coloration is possible, that is another state. Rather than claim more than an inspirational connection with entropy (though there may be), we will use the term flexibility to indicate a known number of coloration states a map may have. We need not compute it exactly, only to show that there exists a flexibility which is not higher than the actual state count of a map. Then if that flexibility is positive the map is colorable.

\section{Definitions and Overview of Approach}

\subsection{Definitions}

The usual convention of representing a planar map as a planar graph, as illustrated in Figure 1, will be adopted. In the terminology of this paper each node (vertex) is a country or region, and the connection (edge) is a shared border. Colors are replaced with substitutable symbols $\mathrm{A}, \mathrm{B}, \mathrm{C}$ and $\mathrm{D}$, which have no fixed color. That is, "A" may be replaced by any one of four colors. Then " $\mathrm{B}$ " simply represents any color different from " $\mathrm{A}$ ", and " $\mathrm{C}$ " any color different from $\mathrm{A}$ and $\mathrm{B}$, and " $\mathrm{D}$ " different from the previous three. Sometimes for economy, when the situation is clear, we will refer to the symbols to identify the node, for example "node A" would be the top node in Figure 1. Ability to label the graph nodes so that no connected nodes have the same color label guarantees the map can be colored. If we redefine the color mapping to the symbols, the map is still colored correctly. Small letters “ $x, y, \ldots$ ” are used as color variables.

Figure 1 shows a fully connected 4-node map. There is no place for a new edge. Ordinary geographical maps, if oceans and lakes are included, will generally be fully connected. But in any case, if a map is fully connected and colorable and an edge is removed, it is still colorable.
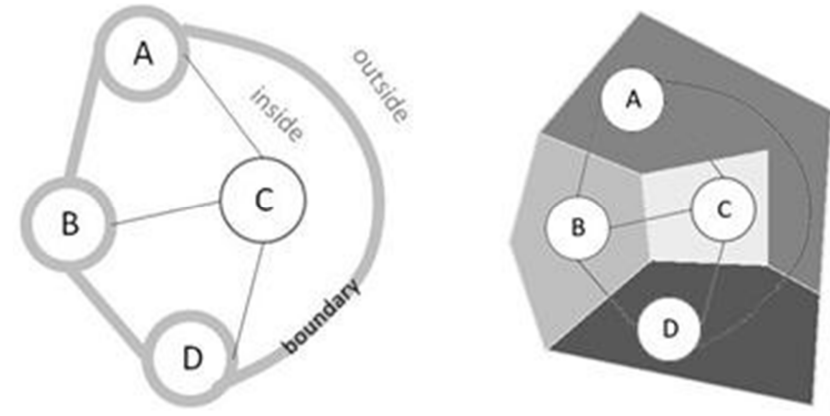

Figure 1. Fully connected 4-node map, represented by a graph on the left, and overlaid with traditional country-area representation on the right.

Figure 1 is also topologically equivalent to all possible fully connected maps of 4 nodes, which we offer without formal demonstration.
Since every one of the 4 nodes is connected to every other, 4 colors are required. Given planarity, one of the nodes must be hidden and only 3 nodes are exposed on the boundary, which we define as those nodes and edges which are exposed to the outside of the map. In this figure, nodes and edges on the boundary are shown very thick for identification, but from here we will use this nomenclature without special graphics. "Boundary" then refers to the map rather than a particular country (node or vertex) which has a "border" or edges. There are several possible boundaries in Figure 1, a matter which we will formally deal with in connection with the order of induction.

\subsection{The Order of Induction}

The induction will begin with a known map of three fully connected nodes which must be contained in any map (as we will show). For less than three nodes there is no apparent outside/inside definable, so the induction would not be uniform.

Using Figure 2 to illustrate terms, the order of induction we will use, which can construct any map, follows:

1. Nodes are added one at a time.

2. Each time a node is added all edges to the existing map are added (one at a time in a separate sub-sequence defined as color flexibility rules).

3. Only one of the innermost nodes outside the current map having more than one connection to the existing map is added (to be explained).

4. Two adjacent edges to the new node are designated as new boundary edges. The old nodes to which they connect are called corner nodes. Any number of interior edges (e.g. $y \rightarrow x$ in Figure 2) may be added, but they will connect to nodes not on the new boundary.

5. The map is redrawn if necessary so that only the two edges, and nodes in between to which the new node does not connect, and the new node itself, are on the new boundary.

6. Any node not on the new boundary is hidden and no new connections from outside can be made.

Some explanation of 3, 4 and 5 is necessary. Figure 2 shows a new node $y$ being added to an existing map. The two dark-thick edges are adjacent with respect to a circumnavigation of the new node $y$. This accomplishes step 4 .

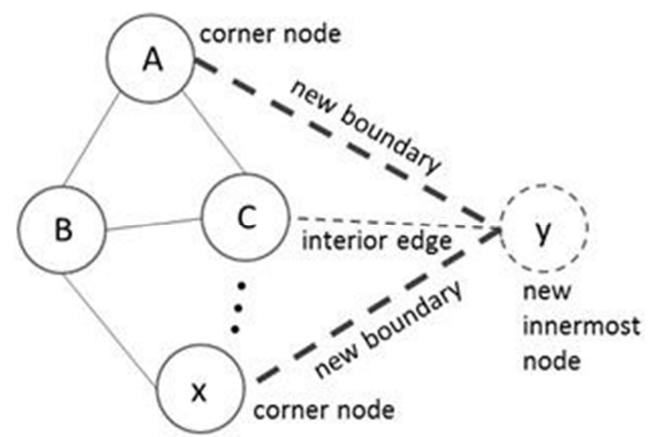

Figure 2. Illustration of adding induction node " $y$ " - solid lines are an existing map, dashed lines are added at the induction step, and ellipses indicates additional similar features. 
The figure could have been drawn with the upper edge looping around the bottom of the map to connect $y$ and A. We must choose either $\mathrm{ABxy}$ or $\mathrm{ACy}$ or $\mathrm{C} \ldots x y$ as the new boundary. Arranging the drawing as in Figure 2 chooses $\mathrm{ABx} y$. In either case, $\mathrm{A}$ and $x$ are the corner nodes for the new addition. Node $y$ will never connect to node $\mathrm{B}$, accomplishing step 5.

Step 3 involves two criteria. Recalling that we require maps to be fully connected, circumnavigate an existing boundary to identify possible new edges, and the outside nodes to which they connect. These all qualify as innermost new nodes. Figure 3 shows an example for identifying possible new edges and their associated nodes. The possible new nodes and edges are shown with dashed lines. We must now show there is at least one node which has, or can have, more than one connection to the existing map in order to satisfy the remaining condition of step 3 .

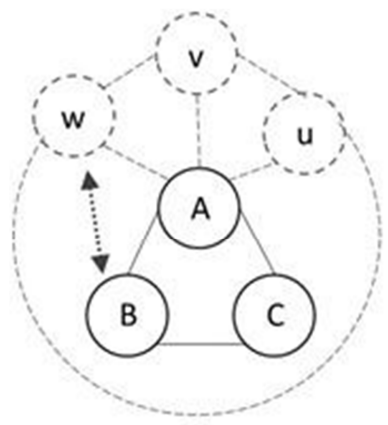

(a)

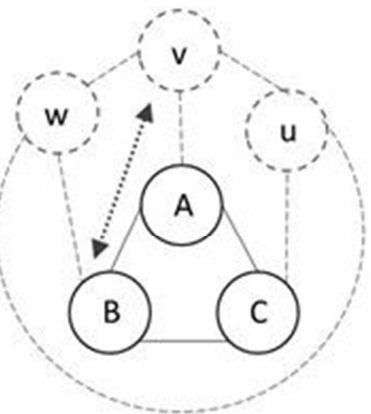

(b)
Figure 3. Examples of identification of possible new edges and nodes (new elements not in existing map are dashed). Presumption is all new structures connecting to existing map (solid lines) are shown. In part (a) several new nodes $w, u, v$ connect to one existing node A, which would create a doubly exposed boundary segment (see Figure 4), however by fully connecting the map (dotted edge with arrows) this is avoided. In part (b) each new node $w, u$, $v$ connects to only one existing node $A, B$ or $C$, so that if connected one at a time again a doubly exposed boundary segment would result. Again by fully connecting the map (dotted double arrow edge) this is avoided. Figure parts (a) and (b) correspond to text cases $a$. and $b$ See text for case $c$.

Consider three cases:

a. There are one or multiple new nodes connecting to the existing boundary (e.g. $u, v, w$ connecting to A in Figure $3 a)$. There are no future connections to the existing boundary as they would have been identified (shown by exclusion circle from $u$ to $w$ ). Since the existing boundary has at least 3 nodes, the map is not fully connected and we can add a connection from a new node to the existing boundary (e.g. dotted arrow $w \rightarrow \mathrm{B}$ ).

b. There are multiple new nodes but each connects to only one existing boundary node (e.g. $u, v, w$ in Figure $3 \mathrm{~b}$ ). Then again the map is incompletely connected and any of the new nodes can connect to one of the existing boundary nodes which connects to its new neighbor (e.g. dotted arrow $v \rightarrow \mathrm{B}$ ).

c. There are multiple new nodes and at least one of them connects to two existing boundary nodes. The condition is immediately satisfied.
Note three things about this procedure. It gives an ordering which can construct any possible fully connected map. Second, during such construction there is always an outside and an inside definable, and new connections are only made to the outside. This expresses the planarity condition. Third, there is never a part of the boundary which is exposed on two sides as illustrated in Figure 4, which would leave $\mathrm{x}$ and $\mathrm{y}$ and the edge between doubly exposed (see cases a and $b$ for prevention). Thus when adding interior edges in step 4 they will never connect to an existing boundary node.

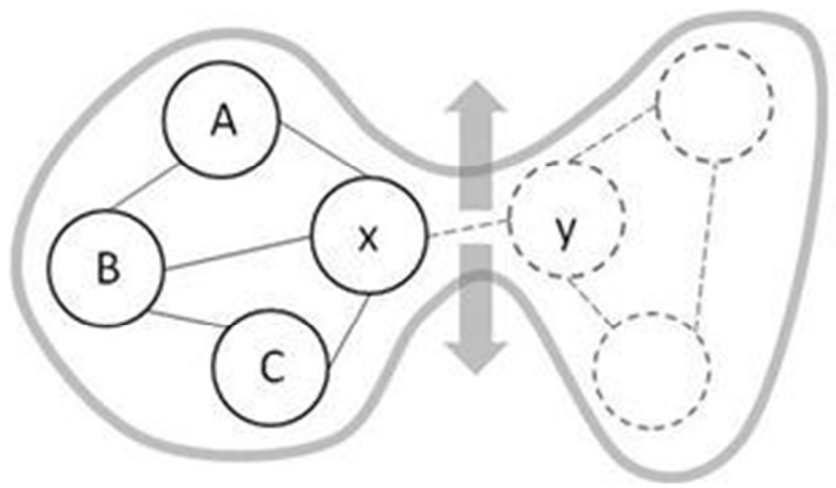

Figure 4. Doubly exposed boundary segment illustration. If node $x$ or $y$ or the edge between were hidden on the top they are still exposed below.

\section{Overview of the induction}

The induction order has been demonstrated to possess two properties related to planarity: the hiding of map sections and avoiding doubly exposed boundary segments. It remains to do the following:

Select a suitable induction hypothesis, a property of the boundary independent of the interior and involving partitioning of the boundary into exposed and hidden parts, which guarantees the map can be colored when a new node is added.

1. Show that the boundary of a universal starter map has this property.

2. Show that the property is true of the boundary when a node is added.

We will have two induction hypotheses, which can be separately proved, theorems 1 and 2 . The first asserts that the whole map flexibility is the product of suitably defined node flexibilities (within the induction sequence), and the second asserts a degree of independence in the guaranteed flexibilities which simplifies computing them, but implies map flexibility is only "at least" that much rather than exactly so.

\section{Starter Map and Color Flexibility}

\subsection{Starter Map}

Suppose we start with one node and add one edge to a second node. This map is fully connected. Using the established induction sequence let us add one new innermost node. By the previously demonstrated principles, this node will connect to both existing nodes forming a 3-node fully connected map. Therefore, every [interesting] map must contain such a starter map which can be used to begin the induction. 


\subsection{Guaranteed Minimum Color Flexibility}

Let us label the first node (in the starter map) with the number of colors from which it may choose. Call this number color flexibility $(F)$. The following initial flexibility-rules will be both amended and justified later:

Start with flexibility $F_{N+1}=4$ for any new node $N+1$.

When adding a new edge, one needs to decrease the color flexibility of only one of the nodes which the edge connects. Either may be chosen.

We assert a physical meaning for guaranteed minimum color flexibility via the following:

Theorem 1: The number of colorations of a map is at least the product of the node flexibilities computed according to the given flexibility-rules.

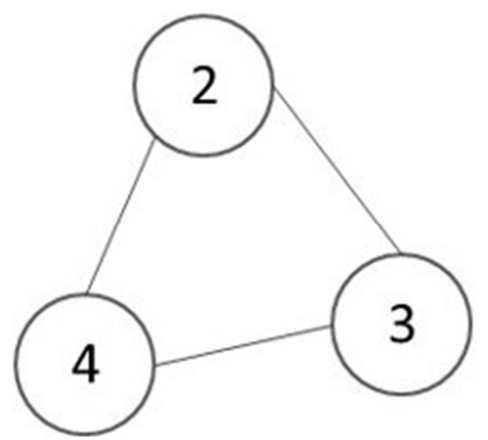

Figure 5. Starter map with color flexibility indicated.

For the starter map, we prove the theorem by enumeration. The combinatorial product of colorations indicated for the 3 -node map in Figure 5 is $F_{\text {map } 3}=4 \times 3 \times 2=24$. The color combinations possible for three nodes 1,2 and 3 are shown in Table 1.

Table 1. Color combinations possible for three nodes.

\begin{tabular}{llll}
\hline & node1 & node2 & node3 \\
\hline 1 & A & B & C \\
2 & A & B & D \\
3 & A & C & B \\
4 & A & C & D \\
5 & A & D & B \\
6 & A & D & C \\
7 & B & A & D \\
8 & B & A & B \\
9 & B & C & A \\
10 & B & C & D \\
11 & B & D & A \\
12 & B & D & C \\
13 & C & A & B \\
14 & C & A & D \\
15 & C & B & A \\
16 & C & B & D \\
17 & C & D & A \\
18 & C & D & B \\
19 & D & A & B \\
20 & D & A & C \\
21 & D & B & A \\
22 & D & B & C \\
23 & D & C & A \\
24 & D & & B \\
\hline
\end{tabular}

\subsection{Flexibility Independence and Revised Rules}

We now introduce the notion independent flexibility whereby a node or group of nodes has a flexibility that is independent of other nodes, so that the flexibilities can be multiplied together to get a minimum map flexibility. The actual map flexibility might be larger. We are only concerned with an easily computable value which is not greater than the actual flexibility. We define the following revised-flexibility-rules for computation on which arguments about independence will be based:

1. For the starter map all boundary nodes have independent flexibility $F_{b} \geq 2$.

2. Assume that for a map of $\mathrm{N}$ nodes all boundary nodes have independent flexibility $F_{b} \geq 2$. Add a new node $\mathrm{N}+1$ according to the induction sequence.

3. When edges are added from new node $\mathrm{N}+1$ to the two corner nodes, decrease the flexibility of node $\mathrm{N}+1$ so that the new node now has independent flexibility $F_{N+1}=2$ and $F_{b} \geq 2$ is preserved in the new map.

4. All further edges will be to newly hidden nodes, formerly on the boundary, but never again subject to edge attachment since there are no double exposed nodes. In those cases decrease the independent flexibility of the newly hidden nodes. Since they previously must have had $F_{b} \geq 2$, and there is never more than a single such connection added to the single new node, none of them will fall below $F_{i}=1$.

These rules avoid the possibility that flexibility ever falls to or below zero for any node. But we have not yet shown the computation is meaningful, which we do now. We extend the notion of independent flexibility to all map boundaries in Theorem 2 below:

Theorem 2: Color flexibilities computed via the stated revised-flexibility-rules and induction sequence from the revised starter map are independent, so that when an edge is placed one possibility can be subtracted from either node connected to the edge, and the map flexibility product still represents a guaranteed minimum number of coloration possibilities.

Back-tracking will be blocked by theorem 2 . That does not mean re-coloring is unnecessary, which can be demonstrated via simple examples. Theorem 2 is abstract and involves conditional facts (flexibility regarding number of choices conditional on color) rather than concrete facts (color).

\section{Induction for Theorems 1 and 2}

The initial state for both theorems has already been proved by enumeration (Table 1), and the induction hypotheses consist of the theorems themselves. For the induction step we assume some existing map with $\mathrm{N}$ nodes and independent flexibility $F_{\text {mapN }}$ which meets the conditions of the theorems, and we add a node $\mathrm{N}+1$ with flexibility $F_{N+1}=4$ and no edges. The assertion of theorem 1 is still true since for every combination of the existing map we can list 4 color choices for 
node $\mathrm{N}+1$ giving $F_{\text {map } N+1}=F_{\text {mapN }} \times 4=F_{\text {mapN }} \times F_{N+1}$. And vice versa, for every color choice of $\mathrm{N}+1$ all the color combinations for the existing map are available, therefore the flexibilities of the existing map and any of its nodes are mutually independent of the flexibility of node $\mathrm{N}+1$, and theorem 2 also remains true.

Now we consider adding an edge from node $\mathrm{M}$ on the border of the existing map with independent flexibility $F_{M}$ to the new node $\mathrm{N}+1$ with independent flexibility $F_{N+1}$, where the existing combined map independent flexibility is $F_{\text {map } N+1}=F_{N+1} \times F_{M} \times F_{\text {mapX } X}$, and where mapX is the map without $\mathrm{M}$ or $\mathrm{N}+1$, and $F_{\text {map } X}$ is the independent flexibility product of all nodes except $\mathrm{M}$ and $\mathrm{N}+1$. Node $\mathrm{M}$ can be either a corner node or a newly hidden interior node. We consider two cases, the first for the independence of choices for node $\mathrm{N}+1$, the second similarly for node $\mathrm{M}$ :

For each coloration possibility of $\operatorname{map} X$ and node $\mathrm{M}$ there are $F_{N+1}$ independent choices for node $\mathrm{N}+1$ (per the pre-existing independence supposition). At most one of them conflicts when an edge is added to node $\mathrm{M}$. Therefore there are at least $F_{N+1}-1$ independent choices for node $\mathrm{N}+1$ which don't conflict with a given configuration of the existing map, and the minimum combined independent flexibility of the new map is at least $F_{\text {map } N+1}=\left(F_{N+1}-1\right) \times F_{M} \times F_{\text {mapX }}$.

For each coloration possibility of $\operatorname{map} X$ and node $\mathrm{N}+1$ there are $F_{M}$ independent choices for node $\mathrm{M}$ (again the pre-existing independence supposition). For each choice at $\mathrm{M}$ at most one of the choices conflicts when an edge is added to node $\mathrm{N}+1$. Therefore there are at least $F_{M}-1$ choices for node $\mathrm{M}$ which don't conflict with the particular configuration of mapX and $\mathrm{N}+1$, and the minimum combined independent flexibility of the new map is at least $F_{\text {map } N+1}=F_{N+1} \times\left(F_{M}-1\right) \times F_{\text {mapX }}$.

The reader may notice that quantities $F_{m a p N+1}$ of case 1 and case 2 are not necessarily equal. It doesn't matter as long as each is not greater than the actual map flexibility.

Now consider adding a second or later edge between node $\mathrm{N}+1$ and the remainder of the map, and again designate the node to which $\mathrm{N}+1$ will connect as being node $\mathrm{Q}$. As the flexibilities are up to now independent, we can decompose the problem of adding an additional edge to make it identical to adding the first edge. Some node somewhere has been decremented to reflect the addition of the first edge. We do not care where except to exclude node Q itself. It cannot in fact have been node $\mathrm{Q}$ because our flexibility computation rules prohibit decrementing non-hidden nodes in the boundary. Thus we can compute a combined map flexibility of $F_{\text {map } N+1}=F_{N+1} \times F_{Q} \times F_{\text {map } Y}$ where $F_{\text {map } Y}$ is a reduced combined flexibility for the map without nodes $\mathrm{Q}$ or $\mathrm{N}+1$, reduced considering the previous edge addition, but not zero or negative since the revised-flexibility-rules will never produce a node flexibility less than one. That is, some node of flexibility 2 or higher has been decremented.
We may now follow the previous argument of cases 1 and 2 using $F_{Q}$ and $F_{\text {map } Y \text {. And we may continue to add edges as }}$ long as each will decrement either the new node (up to two times), or a unique node on the old boundary now hidden. The new map satisfies all necessary conditions for theorems 1 and 2 . If we begin with the revised starter map which has the required independent flexibility $F_{b} \geq 2$ at each boundary node then by induction theorems 1 and 2 are proved, as both the flexibility product and independence properties propagate to every next larger map. The following corollary is evident:

Corollary to Theorem 1: A map is 4-colorable if color flexibility $F_{i} \geq 1$ for $i \in$ all_nodes.

For the induction sequence and the revised-flexibility-rules' choice of decrements, theorems 1 and 2 are true. Thus at each point the flexibilities are independent, and there will never be a case where making a choice at one node decreases the guaranteed minimum number of independent choices at another node. Since the guaranteed minimum flexibility of no non-hidden node (i.e. new boundary node) is decremented by the addition of a new boundary node, and since the new boundary node $\mathrm{N}+1$ has $F_{N+1}=2$, it remains true for the new map of $\mathrm{N}+1$ nodes that for all boundary nodes $F_{b} \geq 2$. Since any fully connected map can be constructed by the induction sequence, then it can be constructed with $F_{i} \geq 1$ meeting the condition of the corollary to theorem 1 . Since any planar map at all can be constructed by removing edges from a fully connected map without decrementing any flexibility assignments, then any planar map is 4-colorable.

\section{Discussion}

A human readable proof has been constructed using the new single-node induction sequence and coloration flexibility concepts. Though some parts were elusive, they seem less elusive in retrospect than other problems long solved. Even Fermat's Last Theorem was proved over a decade ago [7]. It is probably much more difficult (100 pages using mathematics developed long after Fermat) and stood unsolved for twice as long. However, as in the present case, additional time, confidence and new conceptualizations have produced multiple remarkably shorter proofs, and new implications for other fields such as biology [8].

The author has a vague recollection of trying the counting (flexibility) method 40 years ago, but the present induction sequence was not evident to the author at that time. The date of discovery of the induction sequence is uncertain. By then the "human" author was in a repeating loop like an automaton, trying various color exclusion hypotheses, failing because of the uncontrollability of the back-tracking required, eventually forgetting the details but remembering the simplicity, and starting over. Once the induction sequence was in place and color sets were given up in favor of flexibility, the problem was quickly solved in its essentials, though numerous refinements were added over about two years.

The author was not initially familiar with the sub-map ap- 
proaches in the historical literature, and after a brief initial foray into sub-maps quickly abandoned them. Something similar was also a factor in an unrelated century-old problem of finding a simplified derivation for geometric gravity, whose resolution [9] was a stimulus to the present work and results in the following suggestion. The substance of [9] is to decouple exact General Relativity in the observable universe from solutions to the field equations involving exotic topologies which are in some cases unobservable (e.g. beyond the Schwarzschild radius) and in other cases speculative (e.g. wormholes). Thus the author conjectures we might replace the old four-color problem with a new one: that if and only if maps drawn in conventional geometric planes (composed of straight lines, albeit remaining within the spatial geometry) are all four-colorable then the geometry has no wormholes. The effect of a wormhole on coloration is illustrated in Figure 6.

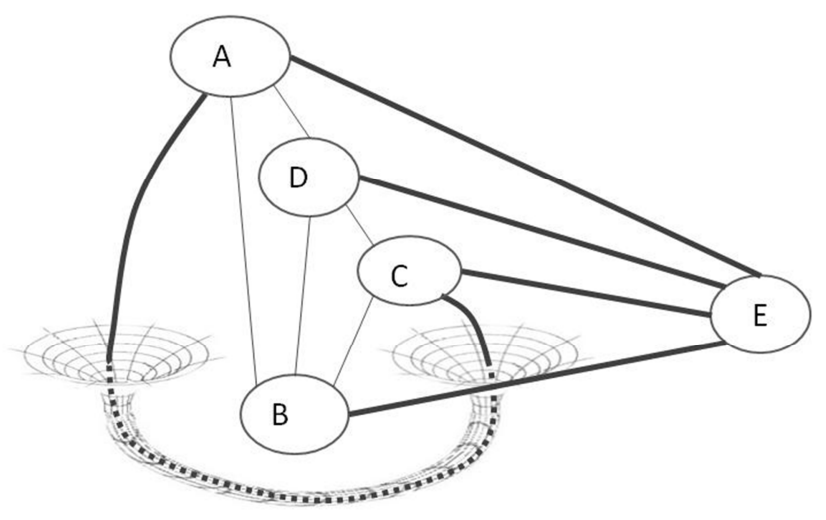

Figure 6. Five color map via wormhole connection.

There is a time version of the conjecture also: that if and only if such maps constructed in time-wise order by the induction sequence are all still 4-colorable then the space-time chronology is protected [10], because connection to a prior point in time would also connect to a since-hidden node and would contradict the premise of 4-color-ability. Defining the "straight lines" suitably is part of the problem. "Geodesics" do not seem appropriate as these can describe photon orbits, which do not seem to have a bearing on map color-ability.

As to physics generally, entropy, and the holographic principle, quantum states are discrete somewhat like color choices, though more complex, and are constrained in many regimes by the Pauli Exclusion Principle. The exclusion principle could be considered analogous to the exclusion of neighboring colors in the map problem. Electrons in an atom, for example, may not have the same energy state as neighbor electrons (in the same atom). This is a very loose analogy, but striking. Counting physical states is a basis of entropy, and leads to the holographic principle in which the information about what has fallen into a black hole is contained on a surface [11]. In the map problem, since in fully connected maps most interior nodes have $F_{i}=1$, the color flexibility as in black hole entropy is a function of the boundary.

There is a limitation of the analogy in the following respect. Our logical maps have the peculiar property that some interior nodes may be rendered irrelevant by enclosing topology. The boundary even shrinks if more than one node is hidden when adding a new boundary node. However, if we allow the boundary nodes to increase in number, then for fully connected maps the coloration flexibility (or entropy) of the entire map increases (even if the number of total nodes does not). The minimum flexibility computed according to our rules does not change unless the number of nodes in the boundary changes, and so is entirely a function of the boundary.

To obtain a concept of mass similar to the surface entropy of a black hole, a different set of connection and hiding rules than in the planar map problem would be required. They should correspond in detail to quantum states, a formidable task somewhat hidden by the problem of quantum gravity in very strong fields. In the meantime, the author suggests exploration of purely mathematical systems having minimum or sufficient connection and state counting properties to gain fundamental understanding. Verlinde has already suggested an entropic origin of gravity emphasizing the holographic principle and thermodynamic entropy [12]. While theoretically equivalent, taking the approach of information entropy and state counting may entail safer or more consistent assumptions. Verlinde finds it necessary to derive inertia from the equivalence principle, which is not entirely safe if space-time "emerges."

Several directions for investigation are evident. Christianto and Smarandache's argument for a discrete-cellular space viewpoint fits well with state-counting models as it eliminates infinitely small points and thus infinitely many. Infinitely many would possibly be ambiguous with respect to counting states [13]. Combining Eddington's notion of a relation between entropy and time [14-15], and the Minkowski-Einstein notion of a relation between space and time [16-17], we find that both space and time must be related to state counting, and beyond a qualitative relationship, it should be productive to pursue a quantitative relationship between state counting and both distance and time. One would expect if the train of thought is followed, it should result in a formulation of distance and time such that if the time or distance (or both) approach zero in one reference frame, the loss of state counts must show up in another reference frame as a maximum and possibly conserved value, with the maximum distance per time value being the universal maximum speed, and negative state counts being prohibited as time travel (also to be understood as reversal of global entropy since time travel enters and accesses the universe at a prior lower entropy point).

If space and time are just state counts, matters of dimensionality arise. Units might be "state counts per state count." Events in space-time would define intervals separated in time or space by (somewhat interchangeable) state counts. Interactions of discrete-cellular space objects (unit masses making quantum state connections with other mass-energy) along a path would enable additional possible states, providing the derivative of summed state counts with respect to path interval state counts. Consider the following as a starting conjecture for investigation:

Gravitational mass is state counts of accumulated mass-energy units, to be formulated by connectivity and hid- 
ing rules that instead of maintaining a state count (flexibility) proportional to boundary nodes only, to the sum of all nodes (mass units), but the states are available at the boundary (analogous to black hole entropy and holographic principle). (We leave questions about reference frames and the progression of nodes beyond the boundary deliberately open.)

Distance (between two events suitably defined) is state counts per unit mass-energy (required to make state interactions).

Time is a change in state counts between two events at a point in space (in effect, entropy increase), again per mass unit.

Velocity is just the usual distance divided by time, but in a state count system this is a unit-less factor with vector direction.

Momentum, which is due to inertial mass in Newton's theory, is then simply gravitational mass state counts times the unit-less velocity vector, and inherently momentum-inertia is linked to the gravitational mass state counts, so equivalence is (or should be) built-in.

Gravity should turn out to be (if the conjectures work out) due to a greater distribution of path-dependent state counts in the direction of mass units available for state-interactions (graph connections with state constraints), so that the probability of state evolution is in the direction of higher density state availability (greater entropy).

Whether or not such a program of investigation produces new fundamental insights, it is likely that a basic view of gravity and space-time in terms of state-counting and probability of moving toward higher state counts may potentially provide an approach to the laws of physical relativity with fewer or different assumptions, which though not easier may be more convincing at lower levels of the educational ladder. If not already, then eventually public resistance to practical limitations on space travel may have adverse impacts on policy and the future of life from Earth. Even physicists complain of the disappointment of giving up their dreams of faster-than-light or wormhole travel. While thermodynamic arguments are obscure except to physicists, and holographic principle arguments obscure to all but specialists, counting arguments are plain even in elementary school. With a few exceptions, gravitational physics has resisted the re-conceptualization process for 100 years since the equivalence principle was used to reason that space-time must "be the field," and for good reason. Most physicists don't trust that the equivalence principle would emerge along with emergent space-time.

Even in Verlinde's approach, space-time as the field is hiding beneath the assumption of equivalence and not really emergent. But the connection to entropy is insightful, and a re-conceptualization along the lines of what happened with both Fermat's Last Theorem and the four color theorem might be accomplished if the state-counting approach can be made uniform with respect to mass-energy itself, so that equivalence emerges exactly. Finally, we must trust that any favorite coordinates, that penetrate spaces unobservable to those unwilling to enter without right of return, will map to the entropy model in a way consistent with thermodynamics, which will distress some. However, they only must map to observable thermodynamics, so there is nothing really new in this except the discomfort of skirting close to well-verified physical law. Some investigators are already arguing the case that real physical gravitational objects would be compatible with reachable state-count spaces [18-19], and even that entropy is a principle agent in effecting this [20].

\section{Conclusion}

At less than six pages for the proof without discussion and research suggestions, and with no difficulty beyond $10^{\text {th }}$ grade geometry, the foregoing appears to qualify as a human readable solution to the four-color problem. In any case, a relevant new conceptualization is presented as a basis for further work.

Additionally it has been shown how the idea of 4-color-ability can be applied in higher dimensions through the use of a plane in those dimensions. If they are regular enough to enforce four-color-ability on such a plane, then likely exotic features are excluded. A second approach to unifying the four color problem with higher dimensional problems is to view them as having a common basis in entropy, or constrained state counting. This might or might not produce additional insights into space-time and gravity, or more accessible approaches. But connecting the entropy argument to the foregoing equivalence of plane color-ability with exclusion of exotic features suggests interesting theoretical arguments.

The difficulty of the seemingly simple four color map problem has led to discussion of the possibility that such problems may not have simple or even human-reachable (without automation) solutions. This may be true. It is not disproved by finding a purely human solution to the four color problem. But it is possible that, like in the case of Fermat's Last Theorem, future changes in conceptualization can impact the accessibility of a difficult problem.

\section{Conflict of Interests Statement and Disclaimer}

The author declares there are no conflicts of interest. The work does not necessarily represent the opinions of the National Aeronautics and Space Administration.

\section{References}

[1] R. Wilson, Four Colors Suffice, Princeton University Press, Princeton 2002, pp 16-19.

[2] K. Appel, and W. Haken, "Every Planar Map is Four Colorable. Part I: Discharging," Illinois Journal of Mathematics, 1977, vol 21 (3) pp 429-490.

[3] Appel, K. and Haken, W. Every Planar Map is Four Colorable. Part II: Reducibility, Illinois Journal of Mathematics, 1977, vol 21 (3) pp 491-567.

[4] E. R. Swart, "The philosophical implications of the four-color problem," American Mathematical Monthly, Mathematical Association of America, 1980, vol 87 (9) pp 697-702. 
[5] N. Robertson, D. P. Sanders, P. Seymour, and R. Thomas, "The Four-Colour Theorem," J. Combin. Theory Ser. B, 1997, vol 70 (1) pp 2-44.

[6] G. Gonthier, "Formal Proof - The Four-color Theorem," Notices of the American Mathematical Society, 2008, vol 55 (11) pp 1382-1393.

[7] A. Wiles, Modular elliptic curves and Fermat's Last Theorem," Annals of Mathematics, 1995, vol 141 (3) pp 448-551.

[8] X. Hong, "A Model-Based Approach for The Demonstration of Fermat's Last Theorem," Pure and Applied Mathematics J., 2017, vol 6 (5) pp 144-147. https://doi.org/ 10.11648/j.pamj.20170605.12.

[9] R. L. Shuler, "A Family of Metric Gravities,” Eur. Phys. J. Plus, 2018, vol 133 pp 158.

[10] S. W. Hawking, "The chronology protection conjecture," Phys. Rev. D, 1992, vol 46 pp 603-611.

[11] L. Susskind, "The World as a Hologram,” J. Mathematical Phys., 1995, vol 36 (11) pp 6377-6396.

[12] E. Verlinde, "On the origin of gravity and the laws of Newton," Journal of High Energy Physics, 2011, vol 29. https://doi.org/10.1007/JHEP04 (2011) 029.

[13] V. Christianto and F. Smarandache, "How many points are there in a line segment? - A new answer from Discrete-Cellular Space viewpoint," J Pur Appl Math., 2018, vol 2 (1) pp 1-4.
[14] A. S. Eddington, The Nature of the Physical World, The Macmillan Co., Basingstoke UK, 1928.

[15] J. L. Lebowitz, "Time's arrow and Boltzmann's entropy," Scholarpedia, 2008, vol 3 (4) pp 3448.

[16] H. Minkowski, Raum und Zeit [Space and Time], Physikalische Zeitschrift, 10: 75-88, 1908-1909. Translation at https://en.wikisource.org/wiki/Translation:Space_and_Time.

[17] A. Einstein [tr. S. N. Bose], The Foundation of the Generalised Theory of Relativity, Annalen der Physik, Vol. 354 (7) pp 769-822,

1916. http://www.archive.org/details/principleofrelat00eins.

[18] C. Chafin, "Globally Causal Solutions for Gravitational Collapse," 2014, arXiv 1402.1524v1 [gr-qc].

[19] J. S. Spivey, "Dispelling Black Hole Pathologies Through Theory and Observation," Progress in Physics, 2015, vol 11 (4) pp 321-329.

[20] T. W. Marshall, "The Shell Collapsar - A Possible Alternative to Black Holes," Entropy, 2016, vol 18 (10) pp 363. http://dx.doi.org/10.3390/e18100363. 